\title{
Delineating the underlying molecular mechanisms and key genes involved in metastasis of colorectal cancer via bioinformatics analysis
}

\author{
CHAO QI $^{1 *}$, YUANLEI CHEN $^{2 *}$, YIXUAN ZHOU $^{1}$, XUCHENG HUANG $^{1}$, \\ GUOLI LI $^{1}$, JIN ZENG ${ }^{1}$, ZHI RUAN ${ }^{1}$, XINYOU XIE ${ }^{1}$ and JUN ZHANG ${ }^{1}$ \\ Departments of ${ }^{1}$ Clinical Laboratory and ${ }^{2}$ Urology, Sir Run Run Shaw Hospital, School of Medicine, \\ Zhejiang University, Hangzhou, Zhejiang 310016, P.R. China
}

Received September 19, 2017; Accepted February 23, 2018

DOI: 10.3892/or.2018.6303

\begin{abstract}
Colorectal cancer (CRC) is considered the world's fourth most deadly cancer. Its metastasis is associated with poor prognosis and weakens the effects of treatment. However, the potential molecular mechanisms and key genes involved in CRC metastasis have remained to be comprehensively elucidated. The objective of the present study was to identify the key genes and molecular pathways underlying CRC metastasis. Differentially expressed genes (DEGs) between primary CRC tissues and metastatic CRC were identified by analyzing the GSE2509 dataset from the Gene Expression Omnibus database. Subsequently, the DEGs were subjected to Gene Ontology (GO) term and Kyoto Encyclopedia of Genes and Genome (KEGG) pathway enrichment analyses via the Database for Annotation, Visualization and Integrated Discovery (DAVID). Next, the top ten hub genes were identified
\end{abstract}

Correspondence to: Professor Jun Zhang, Department of Clinical Laboratory, Sir Run Run Shaw Hospital, School of Medicine, Zhejiang University, 3 Qingchun East Road, Hangzhou, Zhejiang 310016, P.R. China

E-mail: jameszhang2000@zju.edu.cn

${ }^{*}$ Contributed equally

Abbreviations: CRC, colorectal cancer; DEGs, differentially expressed genes; GEO, Gene Expression Omnibus; KEGG, Kyoto Encyclopedia of Genes and Genomes; STRING, Search Tool the Retrieval of Interacting Genes; DAVID, Database for Annotation Visualization and Integrated Discovery; GO, Gene Ontology; PPI, protein-protein interaction; RT-qPCR, reverse transcriptionquantitative polymerase chain reaction; EGFR, epidermal growth factor receptor; HRas, Has proto-oncogene GTPase; Akt1, serine/threonine kinase 1; Wnt5a, Wnt family member 5A; CDKN1a, cyclin-dependent kinase inhibitor 1A; XOR, xanthine oxidoreductase

Key words: colorectal cancer, metastasis, molecular pathways, bioinformatics analysis, microarray, protein-protein interactions in a protein-protein interaction (PPI) network. Sub-network and pathway enrichment analysis were respectively performed with the plugin MCODE and DAVID. Finally, reverse transcription-quantitative polymerase chain reaction assays were performed to corroborate the expression levels of the top five potential metastasis-associated genes in the clinical samples of CRC patients. A total of 7,384 DEGs were obtained, among which 3,949 were upregulated and 3,435 were downregulated. GO and KEGG enrichment analyses identified numerous possible biological processes and pathways that may have a role in the metastasis of CRC. The leading ten hub genes, recognized from the PPI, were epidermal growth factor receptor (EGFR), Has proto-oncogene GTPase (HRas), Wnt family member 5A (Wnt5a), serine/threonine kinase 1 (Akt1), cyclin-dependent kinase inhibitor 1A (CDKN1a), early growth response 1 , Ras homolog family member A, cyclin D1 and Ras-related C3 botulinum toxin substrate 1. Sub-network analysis disclosed the most prominent three modules. Ultimately, EGFR, HRas and Akt1 were verified to be upregulated DEGs, while Wnt5a and CDKN1a were downregulated DEGs when compared with the primary controls. In conclusion, the present study revealed several key genes and relevant molecular mechanisms that may enhance the current understanding of $\mathrm{CRC}$ metastasis, making them significant potential foci for the discovery of further CRC treatments.

\section{Introduction}

Colorectal cancer (CRC), the world's fourth leading cause of cancer-associated mortality, is the third most common type of malignancy in females and the third most common type in males (1-3). At present, $>1$ million patients suffer from CRC, and $>600,000 /$ year succumb to it worldwide. Although CRC may occur at any age, the risk is highest for individuals aged $>50$ years (4).

At present, the standard therapies for CRC are surgical resection, radiotherapy, chemotherapy and targeted therapy $(5,6)$. Metastasis is one of the most critical factors impacting the prognosis of CRC patients and the efficiency of their treatment. Even though the 5-year survival rate for CRC patients without metastasis is high, $\sim 10 \%$ of them gradually 
develop metastasis, which eventually leads to death, rendering the above therapeutic therapies inefficient (7). Furthermore, the majority of patients are diagnosed with $\mathrm{CRC}$ at stages when the cancer cells have further metastasized to other tissues and organs in the body $(8,9)$.

CRC patients tend to develop lymph node metastasis and distant metastasis at late stages. Previous investigations have corroborated that changes in various processes, including increased cell proliferation, altered cell metabolism, decreased apoptosis, activated protease systems take part in the development of adenoma-carcinoma metastasis (10). However, the underlying molecular mechanisms and key genes involved in CRC progression or metastasis have remained to be fully elucidated. Consequently, the exposure of these molecular mechanisms and key genes is the most critical step towards preventing metastasis. In recent years, bioinformatics analysis, including high-throughput sequencing technology and protein-protein interactions (PPIs), has provided a deeper understanding of the aberrant genetic pathways involved in cancers $(11,12)$. In the present study, a bioinformatics analysis was performed to identify the underlying molecular mechanisms and key genes involved in the metastasis of CRC.

Initially, differentially expressed genes (DEGs) between primary CRC tissues and metastatic CRC were identified from datasets downloaded from the Gene Expression Omnibus (GEO) online database. Subsequently, function and pathway enrichment analysis were performed on the DEGs. The ten leading hub genes which may be involved in the CRC progression of metastasis were selected from the PPI network. Finally, tumor tissues from four patients with metastatic CRC were subjected to reverse transcription-quantitative polymerase chain reaction (RT-qPCR) assays to verify these five leading potential metastasis-associated DEGs.

\section{Materials and methods}

Microarray data processing. The GEO dataset GSE2509, including the mRNA expression profile of 3 SW480 and 3 SW620 cell samples, derived from sequencing and subsequent data analysis by Provenzani et al (13), was downloaded from the National Center of Biotechnology Information GEO database (http://www.ncbi.nlm.nih.gov/geo/). The data had been generated using the GPL96 (HG-U133A) platform (Affymetrix Human Genome U133A Array; Affymetrix; Thermo Fisher Scientific, Inc., Waltham, MA, USA). The SW480 cell line originated from a primary rectal gland carcinoma sample and the SW620 cell line was derived one year later from a lymph node metastasis sample of the same patient.

Screening of DEGs. The raw data were uploaded to the Gene-Cloud of Biotechnology Information online laboratory for further analysis (https://www.gcbi.com.cn/gclib/html/index). DEGs were identified between SW480 and SW620. The threshold for the DEGs was defined with a corrected P-value of $<0.05$ and a fold change (FC) of $>1.2$.

Function and pathway enrichment analysis of DEGs. Gene Ontology (GO) analysis is a useful Bioinformatics tool used to annotate information about gene product function and identify characteristic biological attributes by analyzing high-throughput genome data $(14,15)$. The Kyoto Encyclopedia of Genes and Genomes (KEGG) is a tool for the systematic analysis of gene function, performed by linking series of genes with networks of interacting cellular molecules, including complex pathways (16). To facilitate the function and pathway analysis, the DEGs were copied into the Database for Annotation, Visualization and Integrated Discovery (DAVID), to perform the GO and KEGG enrichment analyses. The human genome was chosen as the background list. $\mathrm{P}<0.05$ was considered to indicate a statistically significant difference.

PPInetwork and module analysis. The PPI is described through an undirected diagram with nodes symbolizing the genes and edges symbolizing the mutual interactions of proteins encoded by the corresponding genes (17). In the present study, all of the DEGs were imported into the Search Tool for the Retrieval of Interacting Genes (STRING; http://www.string-db.org) for analysis and only interactions with a combined score of $>0.7$ were pasted into the Cytoscape plugin to create the network visualization. Subsequently, the PPI network was subjected to module analysis by using the Plugin MCODE with the default parameters (Degree cutoff $\geq 2$, Node score cutoff $\geq 2$, $\mathrm{K}$-core $\geq 2$ and Max depth $=100$ ). Finally, function and pathway enrichment analysis of the DEGs in the most prominent three modules were performed via DAVID.

Verification of the leading five potential metastasis-associated DEGs in clinical tumor tissue samples. In the present study, RT-qPCR assays were used to validate the expression levels of the top five potential metastasis-associated genes with the highest degree of interaction and significance in the PPI network. Total RNA was extracted from metastatic and primary CRC samples from four patients who had been diagnosed with metastatic CRC by the Pathology Department of Sir Run Run Shaw Hospital (Hangzhou, China) from October 2016 to March 2017.

First, the mRNA was extracted from the patients' samples using TRIzol reagent (Thermo Fisher Scientific, Inc.) and then stored at $-80^{\circ} \mathrm{C}$. A Reverse Transcription System $\left(\right.$ GoTaq $^{\circledR}$ Real-Time PCR System; Promega Corp., Madison, WI, USA) was employed for the synthesis of complementary DNA according to the manufacturer's instructions. The mRNA expression levels of the key genes were measured by quantitative real-time-PCR using the ABI PRISM 7500 Sequence Detector System (Applied Biosystems; Thermo Fisher Scientific, Inc.), and GAPDH was used as an internal standard. The primers used for qPCR were used as follows: Epidermal growth factor receptor (EGFR) forward, 5'-AGGCACGA GTAACAAGCTCAC-3' and reverse, 5'-ATGAGGACATAA CCAGCCACC-3'; cyclin-dependent kinase inhibitor $1 \mathrm{~A}$ (CDKN1a) forward, 5'-TGTCCGTCAGAACCCATGC-3' and reverse, 5'-AAAGTCGAAGTTCCATCGCTC-3'; Wnt family member 5A (Wnt5a) forward, 5'-TTCTTGGTGGTCGC TAGGT-3' and reverse, 5'-TTCTTTGATGCCTGTCTTCG-3'; Has proto-oncogene GTPase (HRas) forward, 5'-TTTGCCA TCAACAACACCA-3' and reverse, 5'-TCCTGAGCCTGCCG AGAT-3'; serine/threonine kinase 1 (Akt1) forward, 5'-TCCT CCTCAAGAATGATGGCA-3' and reverse, 5'-GTGCGTTC GATGACAGTGGT-3'; GAPDH forward, 5'-AGACAGCCGC ATCTTCTTGT-3' and reverse, 5'-TGATGGCAACAATGTC 
CACT-3'. The reaction protocol included heating for $3 \mathrm{~min}$ at $95^{\circ} \mathrm{C}$, followed by 40 cycles of amplification $\left(5 \mathrm{sec}\right.$ at $95^{\circ} \mathrm{C}$ and $20 \mathrm{sec}$ at $\left.60^{\circ} \mathrm{C}\right)$. qPCR reactions were performed in triplicate. The final results were analyzed via the $2^{-\Delta \Delta \mathrm{Cq}}$ method with normalization to GAPDH (18).

Statistical analysis. PCR results are presented as the mean \pm standard error of the mean $(n=3)$. Differences between the PCR results for the primary and metastatic tumors were determined by the two-tailed unpaired Student's t-test. Prism 4.0 statistical software (GraphPad Inc., La Jolla, CA, USA) was used for statistical analysis. $\mathrm{P}<0.05$ was considered to indicate a statistically significant difference.

\section{Results}

Identification of DEGs between the SW480 and SW620 CRC cell lines. With the aim of exploring the mechanisms underlying CRC metastasis, publicly accessible microarray datasets were retrieved from the GEO database. In total, 3 cell lines each of SW480 and SW620 pertaining to primary and secondary CRC, respectively, were screened for DEGs. The specified criteria, including $\mathrm{FC}>1.2$ and $\mathrm{P}<0.05$, were met for 7,384 genes, including 3,949 upregulated genes and 3,435 downregulated genes. The DEGs were displayed in a heat map (Fig. 1) and a volcano plot (Fig. 2).

GO and KEGG pathway enrichment analysis. The online biological classification tool DAVID was then employed to analyze the functions and pathways of the 7,384 DEGs. GO analysis suggested that the DEGs were fully involved in 873 biological processes with the premise of false discovery rate $<0.05$ and $\mathrm{P}<0.05$. The upregulated DEGs were mainly involved in DNA metabolic processes, responses to DNA damage stimuli, cellular responses to stress and the cell cycle (Fig. 3), while the downregulated DEGs were significantly involved in the Wnt signaling pathway, negative regulation of the canonical Wnt signaling pathway, cell-cell adhesion and negative regulation of apoptotic processes (Fig. 3).

The biological functions of cells are complex processes mediated by numerous molecules and genes. Through KEGG pathway enrichment analysis, the upregulated DEGs were identified to be significantly enriched in purine metabolism, arginine and proline metabolism, the cell cycle and DNA replication, which may be associated with tumor proliferation and migration (Fig. 4). As to the downregulated DEGs, pathways involved in cancer, focal adhesions, adhesion junctions and the Hippo signaling pathway were most significant (Fig. 4).

PPI network and module analysis. To investigate the interactions and acquire the hub genes for potential metastasisassociated DEGs in CRC, all of the DEGs were analysed by using STRING. Subsequently, the genes whose combined score were $>0.7$ revealed close association between genes and were imported into Cytoscape for further analysis. The PPI network contained 954 nodes and 3,326 interactions (data not shown). The ten leading genes defined as the hub genes were as follows: EGFR, HRas, Akt1, Wnt5a, CDKN1a, early growth response 1, Cd44, Ras homolog family member A, cyclin D1 and Ras-related C3 botulinum toxin substrate 1 (data not

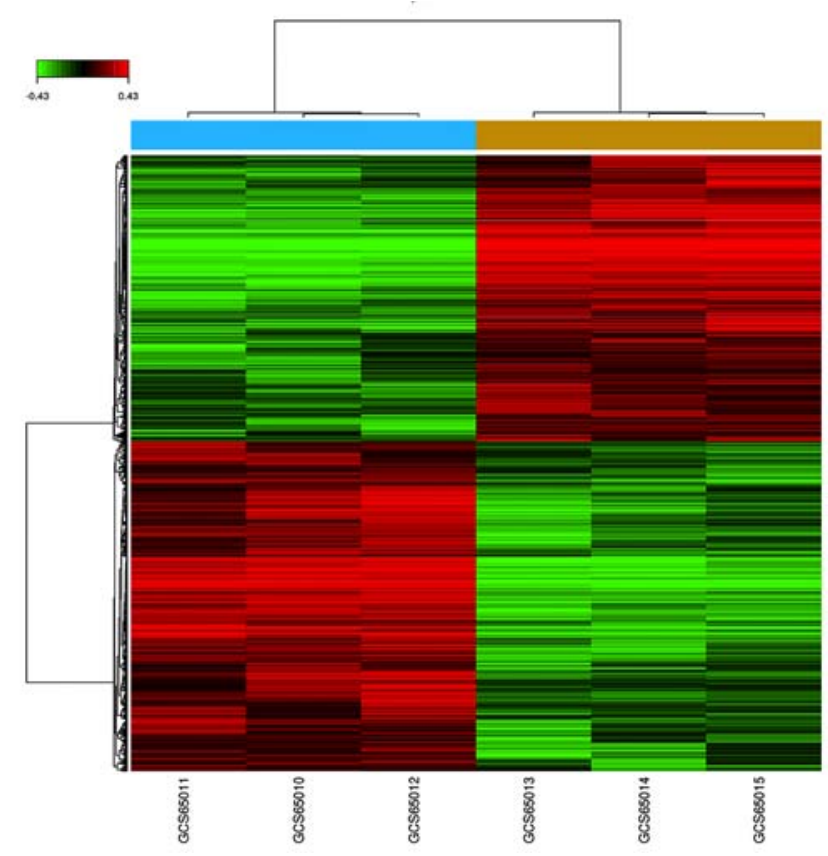

Figure 1. Heat-map of the DEGs in CRC samples versus control samples Blue represented metastatic CRC samples (SW620 cells). Brown demonstrated primary CRC (SW480 cells). Red indicates upregulation and green downregulation. CRC, colorectal cancer; DEGs, differentially expressed genes.

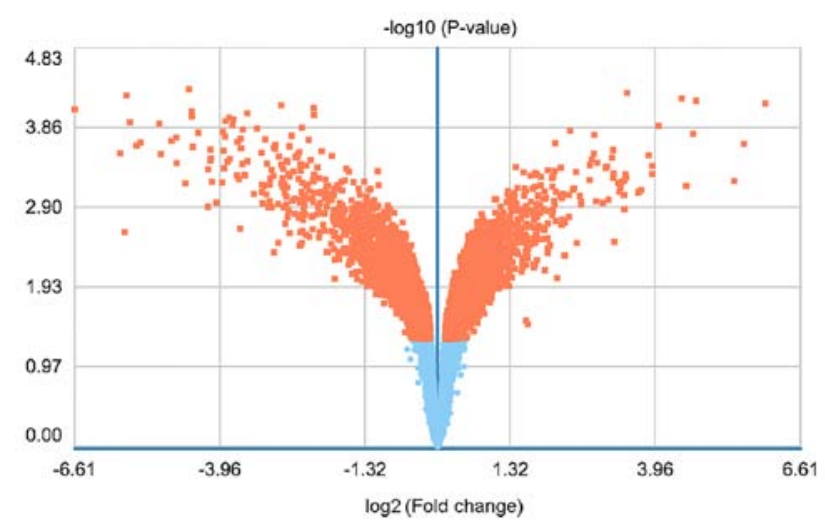

Figure 2. Volcano plot of the DEGs. Horizontal axis: Log2lfold changel vertical axis: $-\log 10$ (P-value). Blue represented genes of $\mathrm{P}<0.05$. Orange demonstrated genes of $\mathrm{P}>0.05$, including DEGs. DEGs, differentially expressed genes.

shown). Next, the Plugin MCODE was employed to recognize the module genes from the PPI analysis (Fig. 5). Function annotation and pathway analysis of the three most significant modules, performed by DAVID, revealed that the module genes were mainly associated with the cell cycle, the forkhead box (FoxO) signaling pathway, the Wnt signaling pathway, ubiquitin-mediated proteolysis, the ERBB2 signaling pathway and the vascular endothelial growth factor (VEGF) signaling pathway (Tables I-III).

Verification of the five most prominent potential metastasis-associated DEGs through analysis of clinical tumor tissue samples. Every DEG analysed with Cytoscape has an interactional degree with others. The five hub genes that had 
Table I. Functional annotation of the significant module 1.

\begin{tabular}{lllr}
\hline Analysis & \multicolumn{1}{c}{ Term } & \multicolumn{1}{c}{ Genes } & \multicolumn{1}{c}{ P-value } \\
\hline GOTERM_BP_DIRECT & $\begin{array}{l}\text { Negative regulation of } \\
\text { epidermal growth factor receptor } \\
\text { signaling pathway }\end{array}$ & AP2S1, CLTA, EGFR & $1.1 \times 10^{-3}$ \\
& ERBB2 signaling pathway & & \\
& Positive regulation of MAPK activity & HRas, STUB1, EGFR & $1.2 \times 10^{-3}$ \\
KEGG PATHWAY & Cell cycle & SKP1, CDC20, CDKN1a & $3.8 \times 10^{-2}$ \\
& FoxO signaling pathway & HRas, CDKN1a, EGFR & $4.2 \times 10^{-2}$ \\
\hline
\end{tabular}

KEGG, Kyoto Encyclopedia of Genes and Genomes; GO, Gene Ontology; BP, biological pro-cesses; MAPK, mitogen-activated protein kinase; FoxO, forkhead box O; EGFR, epidermal growth factor receptor; HRas, Has proto-oncogene GTPase; CDKN1a, cyclin-dependent ki-nase inhibitor 1A; AP2S1, adaptor related protein complex 2 sigma 1 subunit; CLTA, clathrin light chain A; STUB1, STIP1 homology and U-box containing protein 1; CDC20, cell divi-sion cycle 20; SKP1, S-phase kinase associated protein 1.

Table II. Functional annotation of the significant module 2.

\begin{tabular}{|c|c|c|c|}
\hline Analysis & Term & Genes & P-value \\
\hline \multirow[t]{3}{*}{ GOTERM_BP_DIRECT } & $\begin{array}{l}\text { Ubiquitin-dependent } \\
\text { protein cat-abolic process }\end{array}$ & SMURF2, ITCH, RNF6, UBE2C & $1.0 \times 10^{-4}$ \\
\hline & Protein ubiquitination & ASB6, ITCH, KLHL21, UBE2C & $7.4 \times 10^{-4}$ \\
\hline & Wnt signaling pathway & SMURF2, Wnt5A & $4.8 \times 10^{-3}$ \\
\hline \multirow[t]{2}{*}{ KEGG PATHWAY } & Ubiquitin mediated proteolysis & SMURF2, ITCH, RNF6, UBE2C & $2.3 \times 10^{-3}$ \\
\hline & FoxO signaling pathway & SMURF2, ITCH, ARPC1A & $7.9 \times 10^{-3}$ \\
\hline
\end{tabular}

KEGG, Kyoto Encyclopedia of Genes and Genomes; GO, Gene Ontology; BP, biological pro-cesses; FoxO, forkhead box O; Wnt5a, Wnt family member 5A; SMURF2, SMAD specific E3 ubiquitin protein ligase 2; ITCH, itchy E3 ubiquitin protein ligase; RNF6, ring finger protein 6; UBEC2C, ubiquitin-conjugating enzyme E2 C; KLHL21, Kelch-like protein 21; ARPC1A, actin related protein 2/3 complex subunit 1A.

Table III. Functional annotation of the significant module 3.

\begin{tabular}{lll}
\hline Analysis & \multicolumn{1}{c}{ Term } & \multicolumn{1}{c}{ Genes } \\
\hline GOTERM_BP_DIRECT & $\begin{array}{l}\text { Regulation of apoptotic process } \\
\text { Negative regulation of } \\
\text { oxidative stress-induced } \\
\text { intrinsic apoptotic signaling pathway } \\
\text { Positive regulation of blood vessel } \\
\text { endothelial cell migration }\end{array}$ & $\begin{array}{l}\text { Akt1, HSPB1, NOTCH2, PRKCZ } \\
\text { Akt1, HSPB1 }\end{array}$ \\
& Tight junction & Akt1, HSPB1 \\
& VEGF signaling pathway & Akt1, PPKCZ, TJP1 \\
KEGG PATHWAY & Akt1, HSPB1 & $3.6 \times 10^{-3}$ \\
& &
\end{tabular}

KEGG, Kyoto Encyclopedia of Genes and Genomes; GO, Gene Ontology; BP, biological pro-cesses; VEGF, vascular endothelial growth factor; AKT1, serine/threonine kinase 1; HSPB1, heat shock protein family B (small) member 1; PPKCZ, protein kinase C, zeta; TJP1, tight junction protein 1 .

the highest interactional degree of all DEGs were selected as the most prominent potential metastasis-associated DEGs. RT-qPCR analysis was performed to corroborate the expression levels of five potential metastasis-associated genes (EGFR, HRas, Wnt5a, Akt1 and CDKN1a), identified by the above analyses, in four metastatic and four primary CRC samples. EGFR, HRas and Akt1 were upregulated DEGs and their expression levels were identified to be increased, while Wnt5a and CDKN1a were downregulated DEGs and their expression levels were decreased when compared with those in the primary controls. The results of the RT-qPCR analysis are presented in Fig. 6. 
A

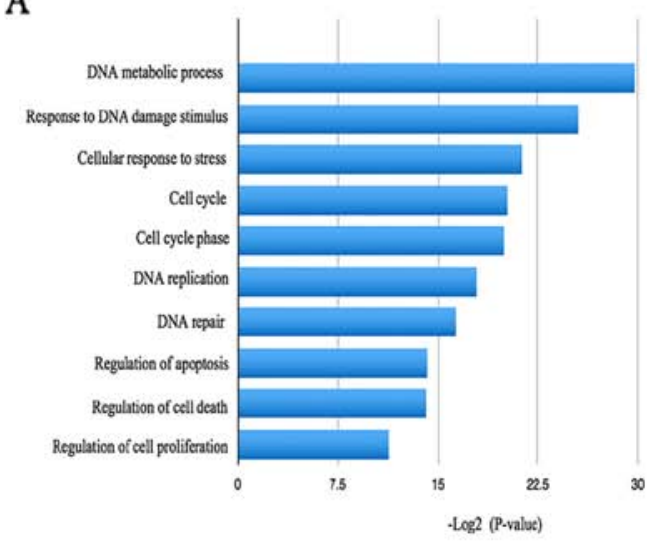

B

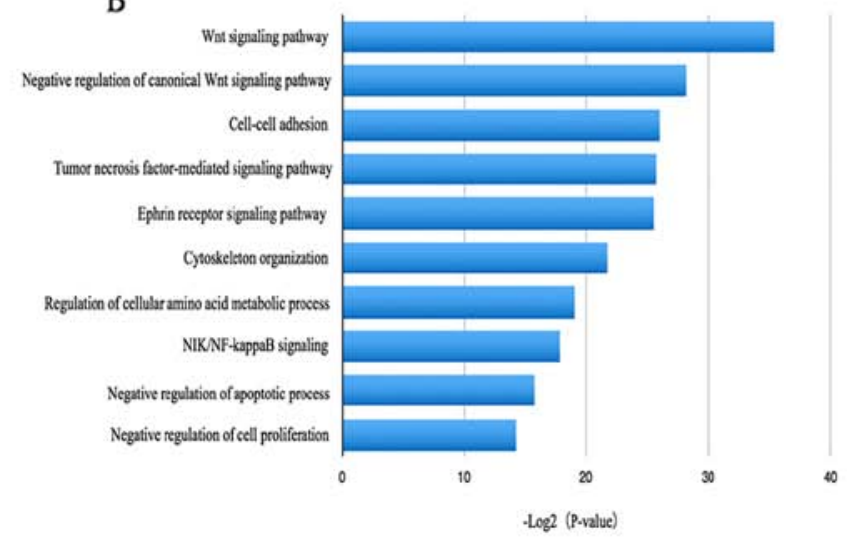

Figure 3. GO analysis of DEGs associated with CRC. GO analysis of (A) upregulated and (B) downregulated genes. GO, Gene Ontology; CRC, colorectal cancer; DEGs, differentially expressed genes.

A

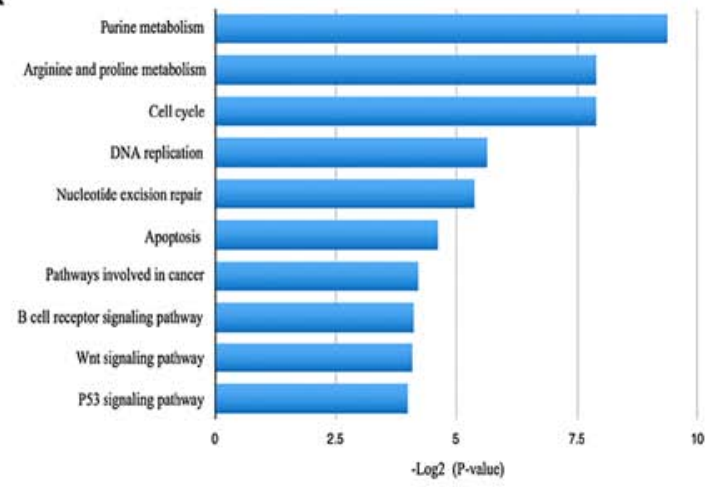

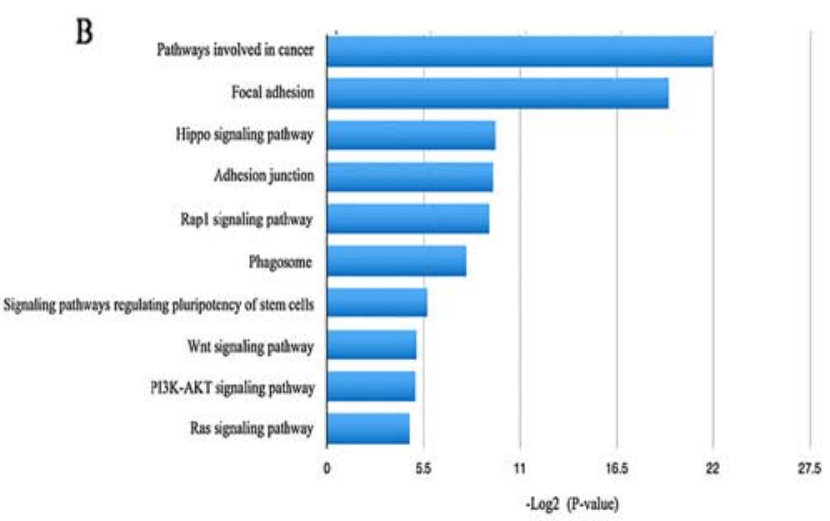

Figure 4. KEGG pathway analysis of DEGs associated with the metastasis of CRC. KEGG pathway analysis of (A) upregulated and (B) downregulated genes. DEGs, differentially expressed genes; CRC, colorectal cancer.

\section{Discussion}

CRC is considered to be a histological heterogeneous disease, and genetic aberrations have a conspicuous role in its occurrence, progression and metastasis $(19,20)$. However, the underlying molecular mechanisms and key genes involved in the progression or metastasis of CRC have remained to be fully elucidated. Consequently, exposure of these molecular mechanisms and key genes is the most critical step in preventing metastasis.

In the present study, 7,384 DEGs, including 3,949 upregulated and 3,435 downregulated genes, were identified from available gene expression profiles. GO and KEGG pathway enrichment analyses subsequently revealed that the DEGs were mainly involved in the cell cycle, regulation of apoptosis, purine metabolism, DNA metabolic process, as well as the Hippo and Wnt signaling pathways.

It is in accord with the current knowledge that the abnormal regulation of apoptosis and the cell cycle are the causes for cancer initiation and progression $(21,22)$.

Uric acid is the final breakdown product in the cycle of nucleotide metabolism. Xanthine oxidoreductase (XOR) acts as a catalyst when uric acid is produced from xanthine (23). Several prior studies revealed that the absence of XOR in tumor cells is significantly associated with poor clinical prognosis and reduced survival rates in breast, stomach, ovarian, colorectal and non-small cell lung cancer (24-26). A recent study, independent of all these variables, revealed that stage IIIA/IIIB CRC patients with high serum uric acid levels may develop early metastasis (27). The present study indicated that DEGs were enriched in purine metabolism, which may correlate with CRC metastasis.

The Hippo pathway participates in the mediation of cell proliferation, differentiation, growth and apoptosis (28). Its negative regulation is often discovered in various types of malignant human tumor, demonstrating that the Hippo signaling pathway is associated with tumor initiation and progression $(29,30)$. In the intestine, the deregulation of the Hippo pathway may lead to neoplastic growth and migration. Furthermore, it has been demonstrated that interactions among the Hippo, Wnt and EGFR signaling pathways have an effect on the development of CRC (31).

In the present study, GO and KEGG pathway enrichment analysis revealed several possible biological processes and pathways, which may be involved in the initiation and development of CRC associated with metastasis.

The results of the module analysis indicated an association of CRC progression with cell cycle activity, negative regulation of the apoptotic process, as well as the Wnt, EGFR, 
A

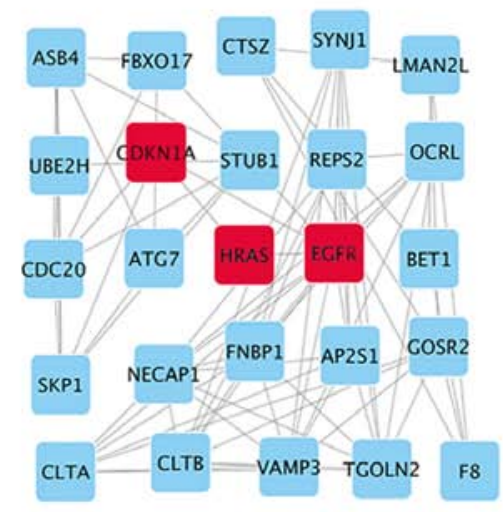

B

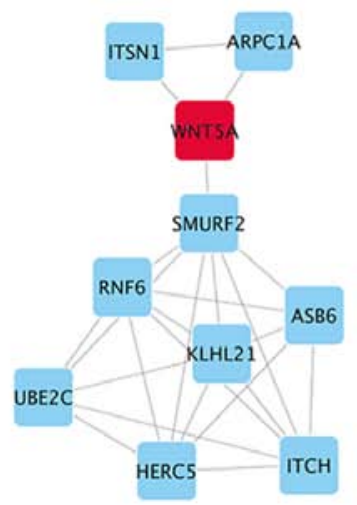

C

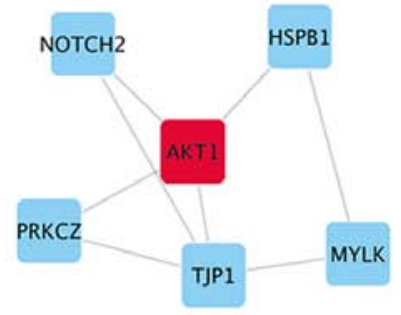

Figure 5. Top 3 modules from analysis of the PPI network. (A) Module 1, (B) module 2 and (C) module 3. EGFR, epidermal growth factor receptor; HRas, Has proto-oncogene GTPase; Akt1, serine/threonine kinase 1; Wnt5a, Wnt family member 5A; CDKN1a, cyclin-dependent kinase inhibitor 1A; ASB4, ankyrin repeat and SOCS box containing 4; FBXO17, F-box protein 17; CTSZ, cathepsin Z; SYNJ1, synaptojanin 1; LMAN2L, lectin, mannose binding 2 like; UBE2H, ubiquitin conjugating enzyme E2 H; STUB1, STIP1 homology and U-box containing protein 1; REPS2, RALBP1 associated Eps domain containing 2; OCRL, inositol polyphosphate-5-phosphatase; ATG7, autophagy related 7; BET1, Bet1 golgi vesicular membrane trafficking protein; SKP1, S-phase kinase associated protein 1; NECAP1, NECAP endocytosis associated 1; FNBP1, formin binding protein 1; AP2S1, adaptor related protein complex 2 sigma 1 subunit; GOSR2, golgi SNAP receptor complex member 2; CLTA, clathrin light chain A; CLTB, clathrin light chain B; VAMP3, vesicle associated membrane protein 3; TGOLN2, trans-golgi network protein 2; F8, coagulation factor VIII; ITSN1, intersectin 1; ARPC1A, actin related protein 2/3 complex subunit 1A; SMURF2, SMAD specific E3 ubiquitin protein ligase 2; RNF6, ring finger protein 6; KLHL21, kelch like family member 21; ASB6, ankyrin repeat and SOCS box containing 6; UBE2C, ubiquitin conjugating enzyme E2 C; HERC5, HECT and RLD domain containing E3 ubiquitin protein ligase 5; ITCH, itchy E3 ubiquitin protein ligase; NOTCH2, notch 2; HSPB1, heat shock protein family B (small) member 1; PRKCZ, protein kinase C $\zeta$; TJP1, tight junction protein 1; MYLK, myosin light chain kinase; PPI, protein-protein interaction.

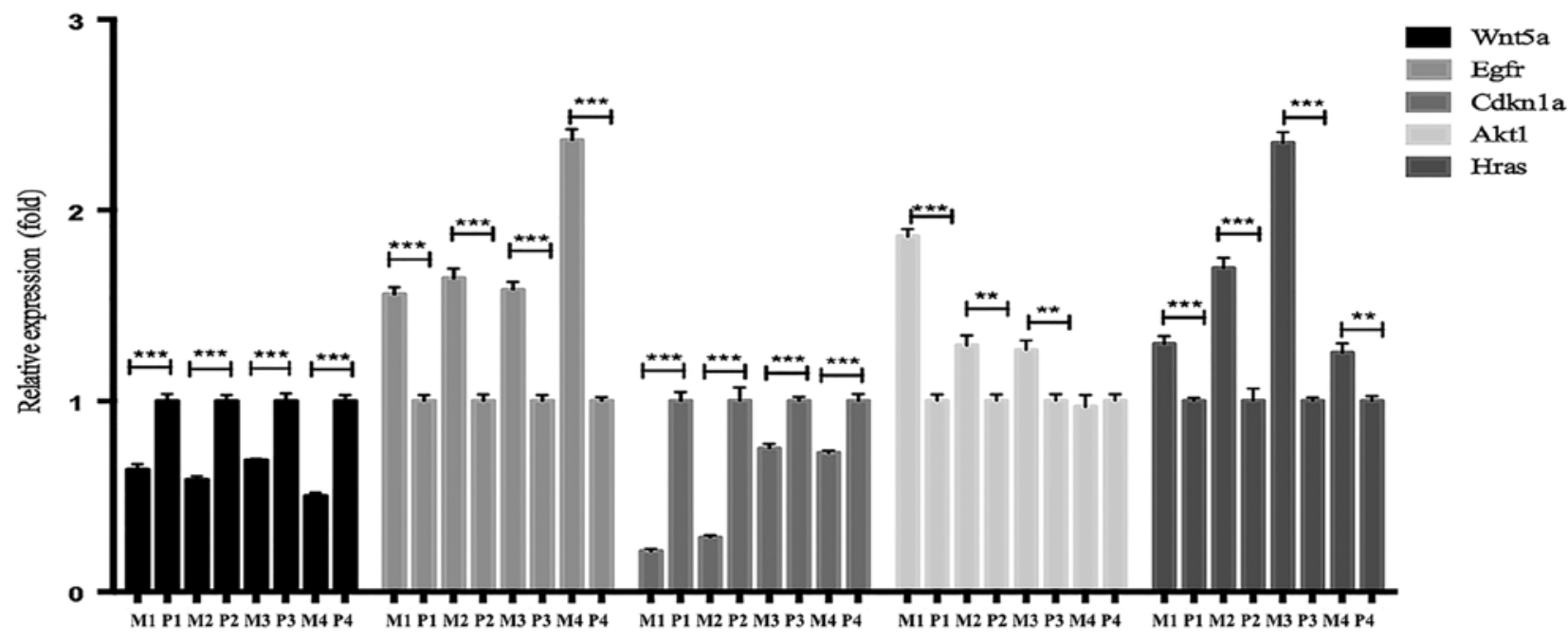

Figure 6. Analysis of the expression levels of hub genes in primary and metastatic clinical CRC samples selected by the interactional degree with other DEGs closely associated with metastasis via Cytoscape. Relative mRNA expression levels were measured by RT-qPCR analysis. ${ }^{* *} \mathrm{P}<0.01 ;{ }^{* * * *} \mathrm{P}<0.001$. P, primary tumor tissues; M, metastatic tumor tissues; EGFR, epidermal growth factor receptor; HRas, Has proto-oncogene GTPase; Akt1, serine/threonine kinase 1; Wnt5a, Wnt family member 5A; CDKN1a, cyclin-dependent kinase inhibitor 1A; CRC, colorectal cancer; DEGs, differentially expressed genes; RT-qPCR, reverse transcription-quantitative polymerase chain reaction.

VEGF and FoxO signaling pathways, which is consistent with the above analyses. In addition, the five leading potential metastasis-associated DEGs (EGFR, HRas, WNT5a, Akt1 and CDKN1a) were involved in the three leading modules.

Wnt5a, located at 3p14.2-p21.1, is known as a non-canonical signaling pathway member of the Wnt family (the Wnt signaling pathway can be classified as canonical Wnt signaling and non-canonical Wnt signaling) (32). Prior studies have observed an elevated expression of Wnt5a in numerous types of cancer and particularly in aggressive tumor cells with greater invasive ability than that of the non-aggressive tumor cells. This delineates the important role of Wnt5a in promoting the progression of tumors through the non-canonical Wnt signaling pathway (33). High expression levels of Wnt5a have been reported to be associated with cancer progression and metastasis in gastric cancer, osteosarcoma and glioblastoma (34-36). Of note, the various functions of Wnt5a rely on disparate cellular contexts in diverse cancer types (33). Wnt5a also inhibits the canonical Wnt signaling pathway (37). In breast cancer, Wnt5a has been reported to inhibit metastasis in vivo, as well as cell migration and invasion in vitro $(38,39)$. In addition, downregulation of Wnt5a is significantly linked 
with the malignant characteristics of primary invasive breast cancer (40). However, Wnt5a was reported to act as a tumor suppressor by obstructing canonical Wnt signaling in CRC (41). Studies focusing on the roles of Wnt5a in the metastatic progression of CRC, determining whether a lower expression of Wnt5a is correlated with a poorer clinical outcome, have been rarely conducted. In the present study, it was discovered that Wnt5a was a downregulated DEG and the expression levels of Wnt5a in primary versus metastatic CRC were varied. GO and KEGG analysis indicated that Wnt5a was significantly enriched in Wnt signaling, which may be the mechanism underlying the higher metastatic rate in CRC patients with decreased expression levels of Wnt5a. Consequently, targeting Wnt5a is likely to be a novel therapeutic strategy for metastatic CRC.

HRas is a member of the Ras superfamily, which comprises KRas, HRas and NRas (42-44). Since HRas mutations do not appear frequently as do mutations in KRas, few studies have been performed with this regard. Several previous studies have reported that HRas directly activates the Ras/BRAF/mitogen-activated protein kinase (MAPK) kinase (MEK)/MAPK and phosphoinositide-3 kinase $(\mathrm{PI} 3 \mathrm{~K}) / \mathrm{Akt} / \mathrm{mammalian}$ target of rapamycin (mTOR) pathway, bypassing EGFR-initiated signaling (45-47). The activation of HRas is likewise associated with galectin-1. The inhibitors of galectin-1, including OTX008, and of mTOR, including rapamycin, may nearly completely suppress Hras-mutant cancers (48). A recent study has revealed that HRas mutations have the tendency to affect patients' outcomes (hazard ratio $=0.545,95 \%$ confidence interval $=0.277-1.073$, $\mathrm{P}=0.079$ ) (49). Furthermore, non-mutated HRas has been identified to be upregulated in CRC cells when compared with normal adjacent tissues. A positive association has been noted between HRas expression levels and the up and downstream signaling factors of EGFR, MEK and extracellular signal-regulated kinase, indicating that HRas overexpression contributes to the carcinogenesis of CRC (50). Despite these studies, HRas expression levels have rarely been assessed with regard to the metastatic progression of $\mathrm{CRC}$, and the connection between HRas and metastasis has remained elusive. In the present study, PPI and module analysis disclosed that HRas is a significant DEG participating in the metastatic progression of CRC. KEGG enrichment analysis revealed that the RAS/BRAF/MEK/MAPK pathway is likely to be an important pathway involved in metastasis. Subsequent RT-qPCR assays performed on tissues of CRC patients further confirmed that high expression levels of HRas were positively associated with CRC metastasis. Although HRas mutations are rare, the upregulation of HRas implies CRC progression. Therefore, the prognostic value of this indication calls for further study.

Proteins of the Akt family, including Akt1, 2 and 3, possess high homology but are expressed by different genes. Akt1, known as an essential molecule in the PI3K/Akt signaling pathway, has a vital role in multiple tumor-associated events, including the promotion of cancer cell proliferation, metabolism and survival $(51,52)$. It is implicated in epithelial-mesenchymal transition and metastasis $(53,54)$. Häggblad Sahlberg et al $(53)$ have confirmed that knockout of Akt1 attenuates metastasis and tumor cell growth by upregulating apoptosis and certain metastasis-inhibitory genes in CRC. In addition, Akt1 directly activates focal adhesion kinase via serine phosphorylation, facilitating the metastasis of pressure-induced CRC (55). In the present study, the PPI network revealed that Akt1 exhibited a high degree of connectivity. In addition, the present KEGG enrichment analysis demonstrated that Akt1 participates in regulating the VEGF signaling pathway, while the latter promotes tumor angiogenesis, development and metastasis $(56,57)$. Of note, previous studies have confirmed that inhibition of Akt1 reduces the secretion of VEGF (58). Furthermore, the upregulated expression levels of Akt1 in certain metastatic versus paired primary CRC samples were confirmed in the present study by RT-qPCR assays, which was consistent with the above analyses. In summary, Aktl is associated with increased cell invasion and motility in CRC. For CRC patients, a high level of Akt1 may herald advanced colorectal tumor progression and unfavorable outcomes.

EGFR may provide cancer cells with self-sufficient growth ability. EGFR has been reported to be overexpressed in $>80 \%$ of all CRC patients (59), and to be correlated with a higher metastatic risk (60). Simultaneously, an increased expression of EGFR is associated with tumor progression and a lessened survival time of patients with metastatic CRC (61). In addition, the RAS/BRAF/MEK/MAPK and the $\mathrm{PI} 3 \mathrm{~K} / \mathrm{Akt} / \mathrm{mTOR}$ pathway, the two major signaling cascades of the EGFR signaling pathway, are activated by phosphorylated EGFR and have an indispensable role in the progression of CRC (10). EGFR-targeting drugs are the first-line therapy and a powerful strategy for treating CRC patients without Ras mutations based on their molecular profiles (62). In the present study, RT-qPCR assays revealed that the expression levels of EGFR were significantly increased in metastatic tissues, implying the possible involvement of EGFR in CRC metastasis.

The CDKN1a gene, which encodes $\mathrm{p} 21$, a protein regulating anti-growth signals, is located on chromosome 6 (6p21.2) (63). The expression levels of CDKN1a, a key intermediary agent of the p53 response, are mediated by p53 in response to innumerable stress-stimulating factors. It then binds to and blocks several CDKs, restraining cell cycle progression at the G1- and S-phase (64-66). Suppression of CDKN1a leads to the transformation of colorectal adenoma into a malignant tumor and likewise accelerates the proliferation and metastasis of CRC cells (67). Furthermore, downregulation of CDKN1a has been confirmed to be associated with venous involvement, as well as lymph node and liver metastasis $(68,69)$. The present study, consistent with the abovementioned studies, implied that CDKN1a may be a key DEG in the metastasis of CRC. Targeting this gene may be a novel therapeutic strategy.

In conclusion, the present study identified several hub genes and key pathways participating in the progression and metastasis of CRC. These efforts may contribute towards an improved understanding of the molecular mechanisms underlying the progression of $\mathrm{CRC}$, and provide potential biomarkers for clinical surveillance and therapy. However, as the total number of cases analyzed in the present study was insufficient, there may be some bias; further molecular biology experiments are required to further confirm the specific functions of these identified genes involved in CRC metastasis in the future. 


\section{Acknowledgements}

Not applicable.

\section{Funding}

The present study was funded by the Natural Science Foundation of China (grant no. 31401005), the Science Foundation of Health Bureau of Zhejiang Province (grant no. 2015KYA143) and the National Natural Science Foundation of China (grant no. 81271914).

\section{Availability of data and materials}

The datasets used and/or analyzed during the present study are available from the corresponding author on reasonable request.

\section{Authors' contributions}

JZ and XX contributed to the concept, design, guidance and review of the article. YC conducted the analysis of bioinformatics data. $\mathrm{CQ}, \mathrm{YZ}, \mathrm{XH}, \mathrm{JZ}, \mathrm{GL}$ and $\mathrm{ZR}$ conducted the experiments, data analysis and writing of the manuscript.

\section{Ethical approval and consent to participate}

The retrospective clinical study was approved by the Ethics Committee of Sir Run Run Shaw Hospital, College of Medicine, Zhejiang University (Hangzhou, China).

\section{Consent for publication}

Anonymity of patient data was protected.

\section{Competing interests}

The authors declare that they have no competing interests.

\section{References}

1. Choi Y, Sateia HF, Peairs KS and Stewart RW: Screening for colorectal cancer. Semin Oncol 44: 34-44, 2017.

2. Manku G, Hueso A, Brimo F, Chan P, Gonzalez-Peramato $P$, Jabado N, Gayden T, Bourgey M, Riazalhosseini Y and Culty M: Changes in the expression profiles of claudins during gonocyte differentiation and in seminomas. Andrology 4: 95-110, 2016.

3. Siegel R, Desantis C and Jemal A: Colorectal cancer statistics, 2014. CA Cancer J Clin 64: 104-117, 2014.

4. Basu A, Seth S, Arora K and Verma M: Evaluating estradiol levels in male patients with colorectal carcinoma. J Clin Diagn Res 9: BC08-BC10, 2015.

5. Ciombor KK, Wu C and Goldberg RM: Recent therapeutic advances in the treatment of colorectal cancer. Annu Rev Med 66: 83-95, 2015.

6. Yu H, Gao G, Jiang L, Guo L, Lin M, Jiao X, Jia W and Huang J: Decreased expression of miR-218 is associated with poor prognosis in patients with colorectal cancer. Int J Clin Exp Pathol 6: 2904-2911, 2013.

7. Zhou C, Cui F, Li J, Wang D, Wei Y, Wu Y, Wang J, Zhu H and Wang S: miR-650 represses high-risk non-metastatic colorectal cancer progression via inhibition of AKT2/GSK3//E-cadherin pathway. Oncotarget 8: 49534-49547, 2017.

8. De Rosa M, Pace U, Rega D, Costabile V, Duraturo F, Izzo P and Delrio P: Genetics, diagnosis and management of colorectal cancer (Review). Oncol Rep 34: 1087-1096, 2015.
9. Yamasaki M, Takemasa I, Komori T, Watanabe S, Sekimoto M, Doki Y, Matsubara K and Monden M: The gene expression profile represents the molecular nature of liver metastasis in colorectal cancer. Int J Oncol 30: 129-138, 2007.

10. Hagland HR, Berg M, Jolma IW, Carlsen A and Søreide K: Molecular pathways and cellular metabolism in colorectal cancer. Dig Surg 30: 12-25, 2013.

11. Gao M, Zhong A, Patel N, Alur C and Vyas D: High throughput RNA sequencing utility for diagnosis and prognosis in colon diseases. World J Gastroenterol 23: 2819-2825, 2017.

12. Serratì S, De Summa S, Pilato B, Petriella D, Lacalamita R, Tommasi S and Pinto R: Next-generation sequencing: Advances and applications in cancer diagnosis. Onco Targets Ther 9: 7355-7365, 2016

13. Provenzani A, Fronza R, Loreni F, Pascale A, Amadio M and Quattrone A: Global alterations in mRNA polysomal recruitment in a cell model of colorectal cancer progression to metastasis. Carcinogenesis 27: 1323-1333, 2006.

14. Gene Ontology Consortium: The Gene Ontology (GO) project in 2006. Nucleic Acids Res 34: D322-D326, 2006.

15. Gene Ontology Consortium: Gene Ontology Consortium: Going forward. Nucleic Acids Res 43 (D1): D1049-D1056, 2015.

16. Kanehisa M and Goto S: KEGG: Kyoto encyclopedia of genes and genomes. Nucleic Acids Res 28(1): 27-30, 2000.

17. Köhler S, Bauer S, Horn D and Robinson PN: Walking the interactome for prioritization of candidate disease genes. Am J Hum Genet 82: 949-958, 2008.

18. Livak KJ and Schmittgen TD: Analysis of relative gene expression data using real-time quantitative PCR and the 2(-Delta Delta C(T)) Method. Methods 25: 402-408, 2001.

19. Aran V, Victorino AP, Thuler LC and Ferreira CG: Colorectal Cancer: Epidemiology, disease mechanisms and interventions to reduce onset and mortality. Clin Colorectal Cancer 15: 195-203, 2016.

20. Taylor-Weiner A, Zack T, O'Donnell E, Guerriero JL, Bernard B, Reddy A, Han GC, AlDubayan S, Amin-Mansour A, Schumacher SE, et al: Genomic evolution and chemoresistance in germ-cell tumours. Nature 540: 114-118, 2016.

21. Chang YT, Tseng HC, Huang CC, Chen YP, Chiang HC and Chou FP: Relative down-regulation of apoptosis and autophagy genes in colorectal cancer. Eur J Clin Invest 41: 84-92, 2011.

22. Perez R, Wu N, Klipfel AA and Beart RW Jr: A better cell cycle target for gene therapy of colorectal cancer: Cyclin G. J Gastrointest Surg 7: 884-889, 2003.

23. Fini MA, Elias A, Johnson RJ and Wright RM: Contribution of uric acid to cancer risk, recurrence, and mortality. Clin Transl Med 1: 16, 2012.

24. Ikegami T, Natsumeda Y and Weber G: Decreased concentration of xanthine dehydrogenase (EC 1.1.1.204) in rat hepatomas. Cancer Res 46: 3838-3841, 1986.

25. Sun AS and Cederbaum AI: Oxidoreductase activities in normal rat liver, tumor-bearing rat liver, and hepatoma HC-252. Cancer Res 40: 4677-4681, 1980.

26. Tanriverdi O, Cokmert S, Oktay E, Pilanci KN, Menekse S, Kocar M, Sen CA, Avci N, Akman T, Ordu C, et al: Prognostic significance of the baseline serum uric acid level in non-small cell lung cancer patients treated with first-line chemotherapy: A study of the Turkish Descriptive Oncological Researches Group. Med Oncol 31: 217, 2014.

27. Cetin AO, Omar M, Calp S, Tunca H, Yimaz N, Ozseker B and Tanriverdi O: Hyperuricemia at the time of diagnosis is a factor for poor prognosis in patients with stage II and III colorectal cancer (uric acid and colorectal cancer). Asian Pac J Cancer Prev 18: 485-490, 2017.

28. Zhao B, Li L, Lei Q and Guan KL: The Hippo-YAP pathway in organ size control and tumorigenesis: An updated version. Genes Dev 24: 862-874, 2010.

29. Harvey KF, Zhang $X$ and Thomas DM: The Hippo pathway and human cancer. Nat Rev Cancer 13: 246-257, 2013.

30. Liu H, Jiang D, Chi F and Zhao B: The Hippo pathway regulates stem cell proliferation, self-renewal, and differentiation. Protein Cell 3: 291-304, 2012.

31. Wierzbicki PM and Rybarczyk A: The Hippo pathway in colorectal cancer. Folia Histochem Cytobiol 53: 105-119, 2015.

32. Serra R, Easter SL, Jiang W and Baxley SE: Wnt5a as an effector of TGF $\beta$ in mammary development and cancer. J Mammary Gland Biol Neoplasia 16: 157-167, 2011.

33. Endo M, Nishita M, Fujii M and Minami Y: Insight into the role of Wnt5a-induced signaling in normal and cancer cells. Int Rev Cell Mol Biol 314: 117-148, 2015. 
34. Hu B, Wang Q, Wang YA, Hua S, Sauvé CG, Ong D, Lan ZD, Chang Q, Ho YW, Monasterio MM, et al: Epigenetic activation of WNT5A drives glioblastoma stem cell differentiation and invasive growth. Cell 167: 1281-1295, 2016.

35. Kurayoshi M, Oue N, Yamamoto H, Kishida M, Inoue A, Asahara T, Yasui W and Kikuchi A: Expression of Wnt-5a is correlated with aggressiveness of gastric cancer by stimulating cell migration and invasion. Cancer Res 66: 10439-10448, 2006.

36. Yong BC, Lu JC, Xie XB, Su Q, Tan PX, Tang QL, Wang J, Huang G, Han J, Xu HW, et al: LDOC1 regulates Wnt5a expression and osteosarcoma cell metastasis and is correlated with the survival of osteosarcoma patients. Tumour Biol 39: 1010428317691188, 2017.

37. Mikels AJ and Nusse R: Purified Wnt5a protein activates or inhibits beta-catenin-TCF signaling depending on receptor context. PLoS Biol 4: e115, 2006.

38. Prasad CP, Chaurasiya SK, Axelsson L and Andersson T: WNT-5A triggers Cdc42 activation leading to an ERK1/2 dependent decrease in MMP9 activity and invasive migration of breast cancer cells. Mol Oncol 7: 870-883, 2013.

39. Säfholm A, Tuomela J, Rosenkvist J, Dejmek J, Härkönen P and Andersson T: The Wnt-5a-derived hexapeptide Foxy-5 inhibits breast cancer metastasis in vivo by targeting cell motility. Clin Cancer Res 14: 6556-6563, 2008.

40. Jönsson M, Dejmek J, Bendahl PO and Andersson T: Loss of Wnt-5a protein is associated with early relapse in invasive ductal breast carcinomas. Cancer Res 62: 409-416, 2002.

41. Ying J, Li H, Yu J, Ng KM, Poon FF, Wong SC, Chan AT, Sung JJ and Tao Q: WNT5A exhibits tumor-suppressive activity through antagonizing the $\mathrm{Wnt} / \beta$-catenin signaling, and is frequently methylated in colorectal cancer. Clin Cancer Res 14: 55-61, 2008

42. Elbjeirami WM and Sughayer MA: KRAS mutations and subtyping in colorectal cancer in Jordanian patients. Oncol Lett 4: 705-710, 2012.

43. Nakano H, Yamamoto F, Neville C, Evans D, Mizuno T and Perucho M: Isolation of transforming sequences of two human lung carcinomas: Structural and functional analysis of the activated c-K-ras oncogenes. Proc Natl Acad Sci USA 81: 71-75, 1984.

44. Santos E, Martin-Zanca D, Reddy EP, Pierotti MA, Della Porta G and Barbacid M: Malignant activation of a K-ras oncogene in lung carcinoma but not in normal tissue of the same patient. Science 223: 661-664, 1984

45. Espada J, Pérez-Moreno M, Braga VM, Rodriguez-Viciana P and Cano A: H-Ras activation promotes cytoplasmic accumulation and phosphoinositide 3-OH kinase association of beta-catenin in epidermal keratinocytes. J Cell Biol 146: 967-980, 1999.

46. Mendoza MC,ErEE and Blenis J: The Ras-ERK and PI3K-mTOR pathways: Cross-talk and compensation. Trends Biochem Sci 36 320-328, 2011

47. Rodriguez-Viciana $\mathrm{P}$, Warne $\mathrm{PH}$, Vanhaesebroeck B, Waterfield MD and Downward J: Activation of phosphoinositide 3-kinase by interaction with Ras and by point mutation. EMBO J 15: 2442-2451, 1996

48. Michael JV, Wurtzel JG and Goldfinger LE: Inhibition of galectin-1 sensitizes HRAS-driven tumor growth to rapamycin treatment. Anticancer Res 36: 5053-5061, 2016.

49. Chang YY, Lin PC, Lin HH, Lin JK, Chen WS, Jiang JK, Yang SH, Liang WY and Chang SC: Mutation spectra of RAS gene family in colorectal cancer. Am J Surg 212: 537-544, 2016

50. Feng J, Hua F, Shuo R, Chongfeng G, Huimian X, Nakajima T, Subao W and Tsuchida N: Upregulation of non-mutated H-ras and its upstream and downstream signaling proteins in colorectal cancer. Oncol Rep 8: 1409-1413, 2001.

51. Riggio M, Perrone MC, Polo ML, Rodriguez MJ, May M, Abba M, Lanari C and Novaro V: AKT1 and AKT2 isoforms play distinct roles during breast cancer progression through the regulation of specific downstream proteins. Sci Rep 7: 44244, 2017.

52. Hofbauer SW, Krenn PW, Piñón Hof bauer J, Pucher S, Asslaber D, Egle A, Hartmann TN and Greil R: The AKT1 isoform plays a dominant role in the survival and chemoresistance of chronic lymphocytic leukaemia cells. Br J Haematol 172: 815-819, 2016.
53. Häggblad Sahlberg S, Mortensen AC, Haglöf J, Engskog MK Arvidsson T, Pettersson C, Glimelius B, Stenerlöw B and Nestor M: Different functions of AKT1 and AKT2 in molecular pathways, cell migration and metabolism in colon cancer cells Int J Oncol 50: 5-14, 2017.

54. Irie HY, Pearline RV, Grueneberg D, Hsia M, Ravichandran P, Kothari N, Natesan S and Brugge JS: Distinct roles of Akt1 and Akt2 in regulating cell migration and epithelial-mesenchymal transition. J Cell Biol 171: 1023-1034, 2005.

55. Wang S and Basson MD: Akt directly regulates focal adhesion kinase through association and serine phosphorylation: Implication for pressure-induced colon cancer metastasis. Am J Physiol Cell Physiol 300: C657-C670, 2011.

56. Papadimitriou K, Rolfo C, Dewaele E, VanDe Wiel M, Van den Brande J, Altintas S, Huizing M, Specenier P and Peeters M: Incorporating anti-VEGF pathway therapy as a continuum of care in metastatic colorectal cancer. Curr Treat Options Oncol 16: 18, 2015

57. Xu WW, Li B, Lam AK, Tsao SW, Law SY, Chan KW, Yuan QJ and Cheung AL: Targeting VEGFR1- and VEGFR2-expressing non-tumor cells is essential for esophageal cancer therapy. Oncotarget 6: 1790-1805, 2015.

58. Wang H, Zhang C, Ning Z, Xu L, Zhu X and Meng Z: Bufalin enhances anti-angiogenic effect of sorafenib via AKT/VEGF signaling. Int J Oncol 48: 1229-1241, 2016.

59. Salomon DS, Brandt R, Ciardiello F and Normanno N: Epidermal growth factor-related peptides and their receptors in human malignancies. Crit Rev Oncol Hematol 19: 183-232, 1995.

60. Mayer A, Takimoto M, Fritz E, Schellander G, Kofler K and Ludwig H: The prognostic significance of proliferating cell nuclear antigen, epidermal growth factor receptor, and mdr gene expression in colorectal cancer. Cancer 71: 2454-2460, 1993.

61. Srivatsa S, Paul MC, Cardone C, Holcmann M, Amberg N, Pathria P, Diamanti MA, Linder M, Timelthaler G, Dienes HP, et al: EGFR in tumor-associated myeloid cells promotes development of colorectal cancer in mice and associates with outcomes of patients. Gastroenterology 153: 178-190, 2017.

62. Bardelli A and Siena S: Molecular mechanisms of resistance to cetuximab and panitumumab in colorectal cancer. J Clin Oncol 28: 1254-1261, 2010.

63. Georgakilas AG, Martin OA and Bonner WM: p21: A two-faced genome guardian. Trends Mol Med 23: 310-319, 2017.

64. el-Deiry WS, Harper JW, O'Connor PM, Velculescu VE, Canman CE, Jackman J, Pietenpol JA, Burrell M, Hill DE and Wang Y,: WAF1/CIP1 is induced in p53-mediated G1 arrest and apoptosis. Cancer Res 54: 1169-1174, 1994.

65. el-Deiry WS, Tokino T, Velculescu VE, Levy DB, Parsons R, Trent JM, Lin D, Mercer WE, Kinzler KW and Vogelstein B: WAF1, a potential mediator of p53 tumor suppression. Cell 75: 817-825, 1993.

66. Harper JW, Adami GR, Wei N, Keyomarsi K and Elledge SJ.: The p21 Cdk-interacting protein Cip1 is a potent inhibitor of G1 cyclin-dependent kinases. Cell 75: 805-816, 1993.

67. Yasui W, Akama Y, Yokozaki H, Semba S, Kudo Y, Shimamoto F and Tahara E: Expression of p21WAF1/CIP1 in colorectal adenomas and adenocarcinomas and its correlation with p53 protein expression. Pathol Int 47: 470-477, 1997.

68. Matsushita K, Kobayashi S, Kato M, Itoh Y, Okuyama K, Sakiyama $S$ and Isono K: Reduced messenger RNA expression level of p21 CIP1 in human colorectal carcinoma tissues and its association with p53 gene mutation. Int J Cancer 69: 259-264, 1996.

69. Schwandner O, Bruch HP and Broll R: Prognostic significance of p21 and p27 protein, apoptosis, clinical and histologic factors in rectal cancer without lymph node metastases. Eur Surg Res 34: 389-396, 2002. 